\title{
La cellule de Langerhans \\ Cellule dendritique de l'épiderme et des muqueuses
}

La cellule de Langerhans est une cellule dendritique de l'épiderme et des épithéliums stratifiés. Dérivée de la lignée monocytaire (et donc originaire de la moelle), elle est l'archétype de la cellule présentant les antigènes aux lymphocytes $T$. Sa localisation explique qu'elle soit en première ligne de la plupart des stimulations antigéniques et des réponses immuno-allergiques. Exprimant la molécule CD4, récepteur du virus HIV, elle est aussi un réservoir potentiel de cet agent.

\section{Daniel Schmitt Colette Dezutter- Dambuyant Marie-Jeanne Staquet Jean Thivolet}

\section{ADRESSE \\ D. Schmitt : directeur de recherche à l'Inserm. C. Dezutter-Dambuyant: chargée de recherche à l'Inserm. M.-J. Staquet: chargée de recherche à l'Inserm. J. Thivolet : professeur de clinique dermatologique. Directeur de l'U 209 de l'Inserm. Inserm U 209, laboratoire de recherche dermatologique et immunologi- que, hôpital Édouard-Herriot, 69437 Lyon Cedex 03, France.}

\section{TIRÉS A PART}

D. Schmitt.

$m / s n^{\circ} 2$ vol. 5 , février 89

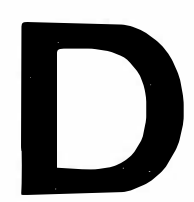

écrite en 1868 par Paul Langerhans, la cellule épidermique qui porte son nom est une cellule dendritique, libre, localisée dans la partie moyenne et profonde de l'épiderme (figures 1 et 2, p. 104 et 105). On la rencontre dans l'ensemble du revêtement cutané (avec des densités variables) et dans les épithéliums muqueux (bouche, vagin, rectum, vessie, poumon). Il en existe un faible pourcentage dans les ganglions. D'origine médullaire, la cellule de Langerhans (CL) présente des organites cytoplasmiques spécifiques appelés granules de Birbeck. Elle exprime, au niveau de sa membrane, différents groupes d'antigènes $(\mathrm{CDl}$, CD4, HLA de classe I et classe II principalement). Fonctionnellement, les cellules de Langerhans agissent comme des cellules présentant les antigènes aux lymphocytes et induisent une réponse immunitaire de type cellulaire. En pathologie cutanée, les cellules de Langerhans interviennent dans les dermites de contact, les réactions de rejet de greffes, les réactions inflammatoires, certaines proliférations ainsi que dans certaines maladies virales parmi lesquelles le SIDA.

\section{Origine et précurseurs des cellules de Langerhans}

Depuis la démonstration chez la souris de l'origine médullaire des cellules de Langerhans par des greffes de moelle allogéniques [2] les mêmes données ont été obtenues chez l'homme après transplantation de moelle dans les cas de donneur et receveur allogéniques et/ou de sexes différents [3].

La caractérisation des précurseurs médullaires des cellules de Langerhans s'est appuyée sur deux notions [4] : l'existence de similitudes entre cellules de Langerhans et monocytes et l'expression des molécules $\mathrm{CDl}$ par un très faible pourcentage de cellules médullaires. De plus, grâce à la possibilité de cultiver les cellules promonocytaires de la moelle et d'identifier l'antigène $\mathrm{CDI}$, il a été possible de décrire les aspects morphologiques et le phénotype des cellules $\mathrm{CDl}+$ de la moelle [5] : ces cellules montrent les aspects des promonocytes/monocytes et n'ont pas de granules de Birbeck. Dans cette 


\section{RÉFÉRENCES}

1. Staquet MJ. Origin and precursors of Langerhans cells. In: Thivolet J, Schmitt D, eds. The Langerhans Cell. Paris : Inserm/ John Libbey Eurotext, 1988 : 9-19.

2. Katz SI, Tamaki K, Sachs DH. Epidermal Langerhans cells are derivated from cells originating in bone marrow. Nature $1979 ; 282$ : 324-6.

3. Perreault C, Pelletier M, Landry D, Gyger M. Study of Langerhans cells after allogeneic bone marrow transplantation. Blood 1984 63 : 807-11.

4. Gothelf Y, Sharon N, Gazit E. Fractionation of human bone marrow mononuclear cells with Peanut Agglutinin: phenotypic characterization with monoclonal antibodies Human Immunol $1986 ; 17: 37-44$

5. De Fraissinette A, Schmitt D, DezutterDambuyant C, Guyotat D, Zabot MT, Thivolet J. Culture of putative Langerhans cell bone marrow precursors : characterization of their phenotype. Exp Hematol $1988 ; 16: 764$ 8.

6. Dezutter-Dambuyant C, Schmitt D, Faure M, Cordier C, Thivolet J. Detection of OKT6positive cells in peripheral blood (without visible Birbeck granules). Immunol Letters $1988 ; 8: 121-6$.

7. Czernielewski J, Vaigot $\mathbf{P}$, Prunieras $M$. Epidermal Langerhans cells : a cycling population. J Invest Dermatol 1985 ; 84 : 424-6.

8. Dezutter-Dambuyant C. Membrane antigens and Birbeck granules of human Langerhans cells. In : Thivolet J, Schmitt D, eds The Langerhans Cell. Inserm/John Libbey Eurotext, 1988; 87-97.

9. Stingl G, Katz SI, Shevach EM, WolffSchreiner E, Green I. Detection of Ia antigens on Langerhans cell in guinea-pig skin.J Immunol 1978 ; 120 : 570-8.

10. Bruynzeel-Koomen C, Bruynzeel P. Inhalant allergens as contactants in patients with atopic dermatitis. J Dermatol 1987 ; $14: 524$.

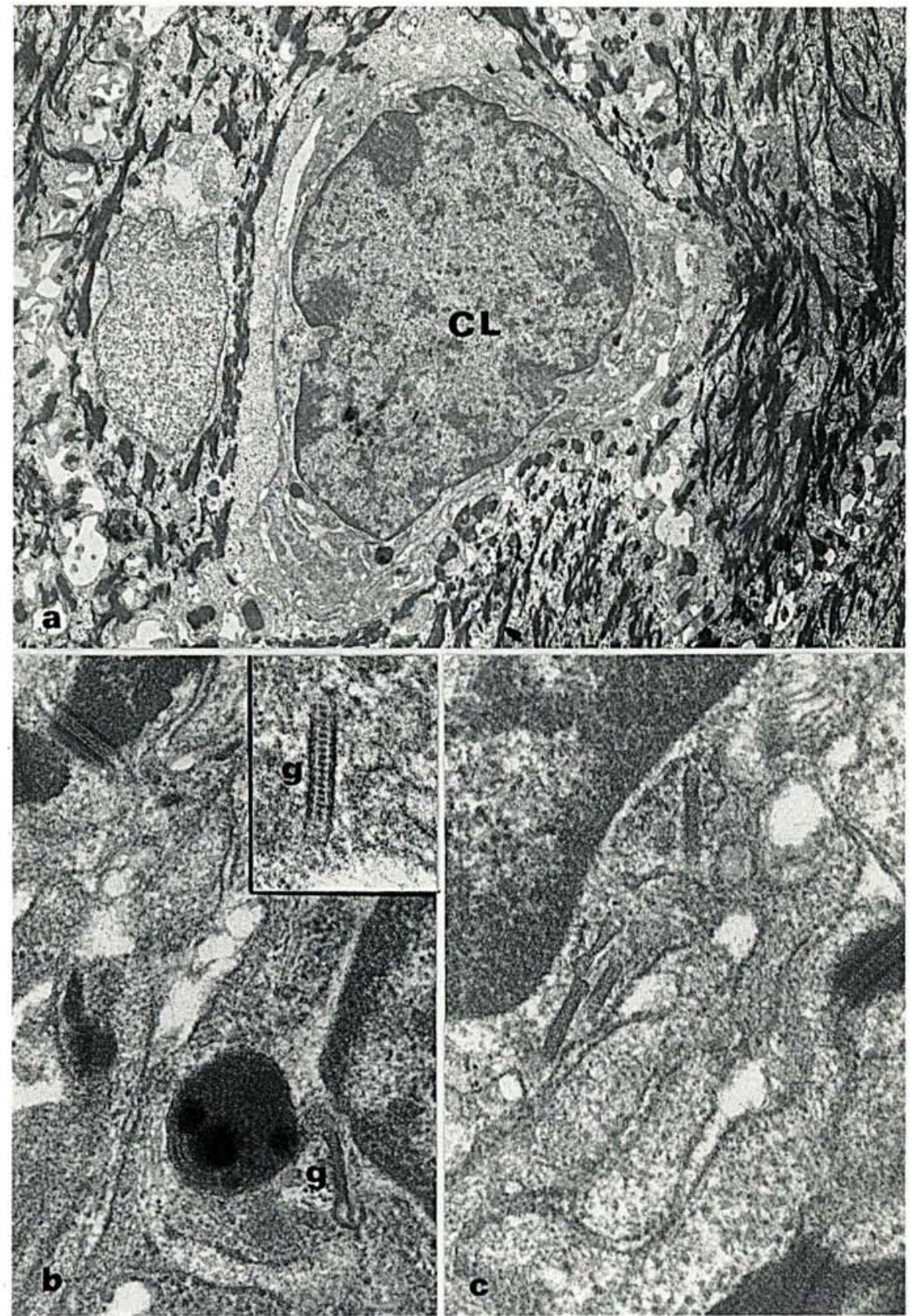

Figure 1. Cellule de Langerhans (CL) in situ au sein des kératinocytes dans l'épiderme humain(a). On note au niveau du cytoplasme des inclusions denses (lysosomes) (b) et des formations spécifiques, les granules de Birbeck (g), qui apparaissent soit sous forme de bâtonnets, soit sous forme de raquettes (b) (c). $(a \times 12000$, b et $c \times 45000$, encart $\times 95000)$.

population cellulaire, les antigènes CD14 et CD33 sont exprimés (marqueurs monocytaires) alors que les antigènes de diférenciation des thymocytes (autres que CDl+) $\mathrm{n}_{\mathrm{e}}$ sont pas retrouvés.

Il n'a pas encore été possible d'induire in vitro la formation par ces cellules médullaires de granules de Birbeck typiques. Cette étape permet- tra alors d'affirmer que ces cellules $\mathrm{CDI}+$ de la moelle sont bien les précurseurs des cellules de Langerhans.

Des cellules de type monocytaire $\mathrm{CDl}+$ ont également été décrites au niveau du sang périphérique, suggérant, entre la moelle et la peau, l'existence d'un précurseur circulant [6]. Dans le sang de cordon, des cellules 
$\mathrm{CDl}+, \mathrm{CDl} 1+, \mathrm{CDl} 4+$ et HLA$\mathrm{DR}+$ ont été décrites. De telles cellules marquées par un anticorps anti-CDl (OKT6) forment des structures ressemblant à des granules de Birbeck. Le pourcentrage de cellules $\mathrm{CDl}+$ circulantes diminue avec l'âge, augmente chez les brûlés et dans les cas de SIDA. Bien qu'on ne puisse pas négliger l'existence des cellules thymocytaires $\mathrm{CDl}+$ circulantes, la sous-population de type monocytaire représente probablement la forme circulante des précurseurs des cellules de Langerhans épidermiques.

Parallèlement au flux de cellules médullaires migrant vers la moelle, le maintien de la population des cellules de Langerhans épidermiques est également assuré par une faible mais réelle capacité de division par mitose des cellules de Langerhans au sein de l'épiderme [7]. L'utilisation de la thymidine tritiée ou de la bromodéoxyuridine $(\mathrm{BrDu})$ montre que, dans les conditions normales, 2 à $6 \%$ des cellules de Langerhans épidermiques sont en phase S. Enfin des images de mitoses ont été observées dans des suspensions de cellules épidermiques fortement enrichies en cellules de Langerhans (50 à $80 \%$ ) : mitoses de cellules $\mathrm{CDl}+$ (Ac DMCl) avec granules de Birbeck (données non publiées).

Dans le cadre de la différenciation des cellules de Langerhans, l'induction de granules de Birbeck dans des cellules médullaires $\mathrm{CDl}+$ au sein d'un environnement épithélial in vitro reste le chaînon manquant des données actuellement recueillies.

\section{Antigènes de membrane des cellules de Langerhans}

A l'exception des activités enzymatiques membranaires (ATPase) ou cytoplasmiques (estérases), les marqueurs immunologiques des cellules de Langerhans sont essentiellement des protéines de surface [8, 9] (figures 3 et 4, p. 106 et 107).

Quatre groupes ont été identifiés : molécules HLA de classe I $(\mathrm{A}, \mathrm{B}, \mathrm{C})$ et de classe II (DP DQ DR), antigènes CDl (CDla et CDlc) et antigènes CD4. Deux populations de cellules de Langerhans normales peuvent être définies sur la base de la $\mathrm{m} / \mathrm{s} n^{\circ} 2$ vol. 5. féurier 89

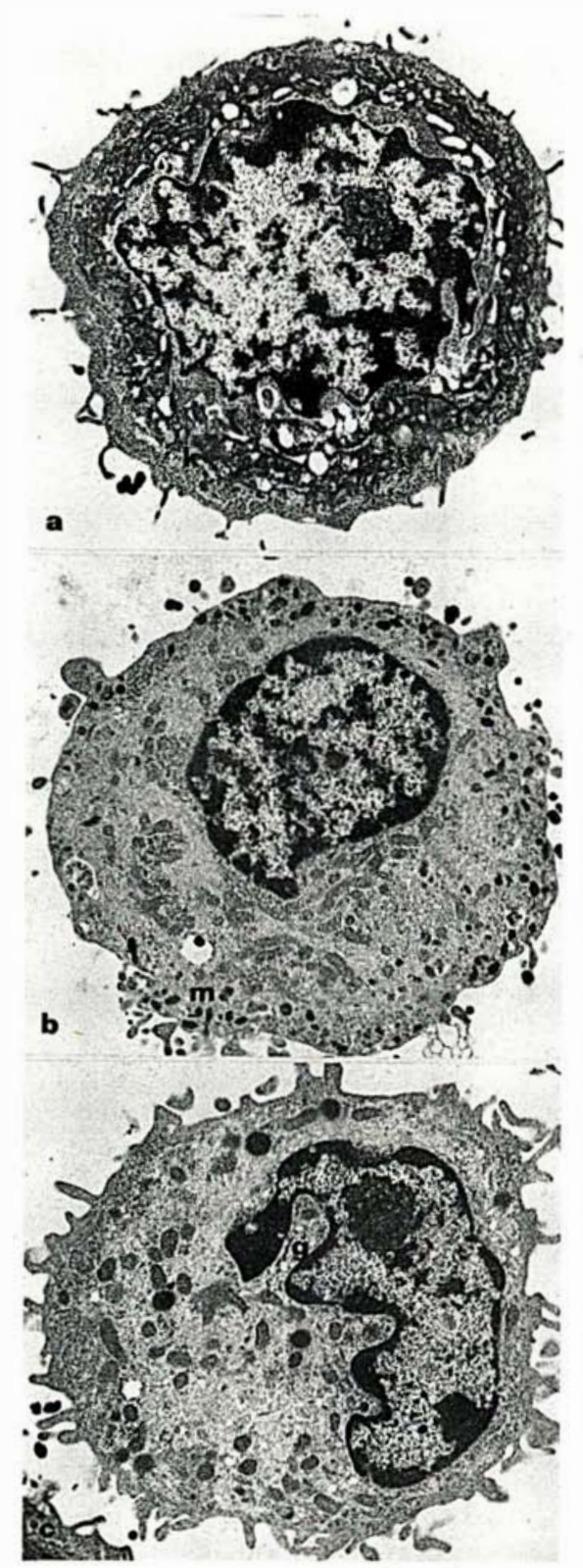

densité des sites DR (25\% de CL $\mathrm{DR}+++$ et $75 \%$ de CL DR+). La densité de surface des molécules de classe II peut être modifiée par les ultraviolets, les stéroïdes, l'interféron $\gamma$ et les prostaglandines E2.

Il existe également, à la surface des cellules de Langerhans, des récepteurs pour le fragment Fc des immunoglobulines $G(\operatorname{IgG})$, pour les fractions C3b et C4d du complément (CD35).

Récemment, diverses molécules ont été mises en évidence à la surface des cellules de Langerhans : marqueurs monocytaires (CDl4, CD33), molécules d'adhésion : chaîne $\beta$ des LFA (leucocyte associated antigen) (CDll, CD18), VLA (marqueurs qui apparaissent à la surface des lymphocytes very late after activation) (CDw29) et, dans la dermatite atopique, des récepteurs pour les immunoglobulines $\mathrm{E}(\mathrm{IgE})(\mathrm{CD} 23)$ [10].

Les molécules CDla (49 $000 \mathrm{Da})(\mathrm{Da}$ $=$ daltons) et CDlc (43000 Da) ont été isolées à partir de la membrane des cellules de Langerhans normales. La molécule de 49000 Da est sensible à la trypsine et donne un produit de dégradation de $27000 \mathrm{Da}$ reconnu par certains anticorps monoclonaux anti-CDla [14]. L'anticorps monoclonal DMCl (Biosoft) ne réagit qu'avec la protéine native de $49000 \mathrm{Da}$.

Les molécules CD4 exprimées par les cellules de Langerhans [12] représentent également le récepteur du virus HIV responsable du SIDA. Cette molécule est sensible à l'interféron $\gamma$ et semble être exprimée très tôt au cours de la différenciation des cellules de Langerhans puisqu'on la trouve déjà sur les promonocytes médullaires.

Les molécules HLA de classe I sont exprimées par les cellules de Langerhans comme par toutes les autres cellules vivantes de l'épiderme. En revanche, la densité de ces antigènes est très faible (environ dix fois moins qu'au niveau des kératinocytes) et ils sont (avec les données quantitatives actuelles) les plus faiblement exprimés des antigènes de surface des cellules de Langerhans, alors que les antigènes $\mathrm{CDl}$ sont quantitativement les plus denses [13].

A l'exception des molécules HLA$\mathrm{DR}$, le rôle de tous ces antigènes de 


\section{RÉFÉRENCES}

11. Dezutter-Dambuyant C, Schmitt D, Staquet MJ, Thivolet J. Biochemical analysis of CDla and CDlc antigens on normal human epidermal Langerhans cells. In: Thivolet J, Schmitt D, eds. The Langerhans Cell, Inserm/ John Libbey Eurotext, 1988 : 125-37.

12. Wood GS, Warner NL, Warnke RA. AntiLeu/T4 antibodies react with cells of monocytic/macrophage and Langerhans cell lineage. J Immunol 1983 ; 131 : 212-6.

13. Gielen V, Schmitt D, Thivolet J. HLA class I antigen (heavy and light chain) expression by Langerhans cells and keratinocytes of the normal human epidermis : ultrastructural quantitation using immunogold labeling Arch Dermatol Res 1988 ; 280 : 131-6.

14. Hanau D, Fabre M, Schmitt DA et al. Human epidermal Langerhans cells cointernalize by « receptor-mediated endocytosis * non-classical major histocompatibility complex class I molecules (T6 antigens) and class II molecules (HLA-DR antigens). Proc Natl Acad Sci USA 1987; $84: 2901-5$.

15. Ray A, Schmitt D, Dezutter-Dambuyant C, Farges MC, Thivolet J. Reappearance of CDla antigenic sites after endocytosis on human Langerhans cells evidenced by immunogold relabelling. J Invest Dermatol 1989 (sous presse).

16. Birbeck MS, Breathnach AS, Everall JD. An ultrastructural study of basal melanocytes and high-level clear cells (Langerhans cells) in vitiligo. J Invest Dermatol 1961; 37 : 5164.

17. Hanau D, Fabre M, Schmitt DA, Garaud JC, Pauly G, Cazenave JP. Appearance of Birbeck-like structures in anti-T6 antibody treated human epidermal Langerhans cells. $J$ Invest Dermatol 1988; 90 : 298-304.

18. Katz SI, Cooper KD, Iijima M, Tsuchida $\mathrm{T}$. The role of Langerhans cells in antigen presentation. J Invest Dermatol 1985 ; 85 : 96s-

19. Faure M. Langerhans cells and antigen presentation. In : Thivolet J, Schmitt D, eds The Langerhans Cell. Inserm/John Libbey Eurotext 1988, 295-9.

20. Braathen LR, Thorsby E. Studies on human epidermal Langerhans cells. I. Alloactivating and antigen presenting capa-
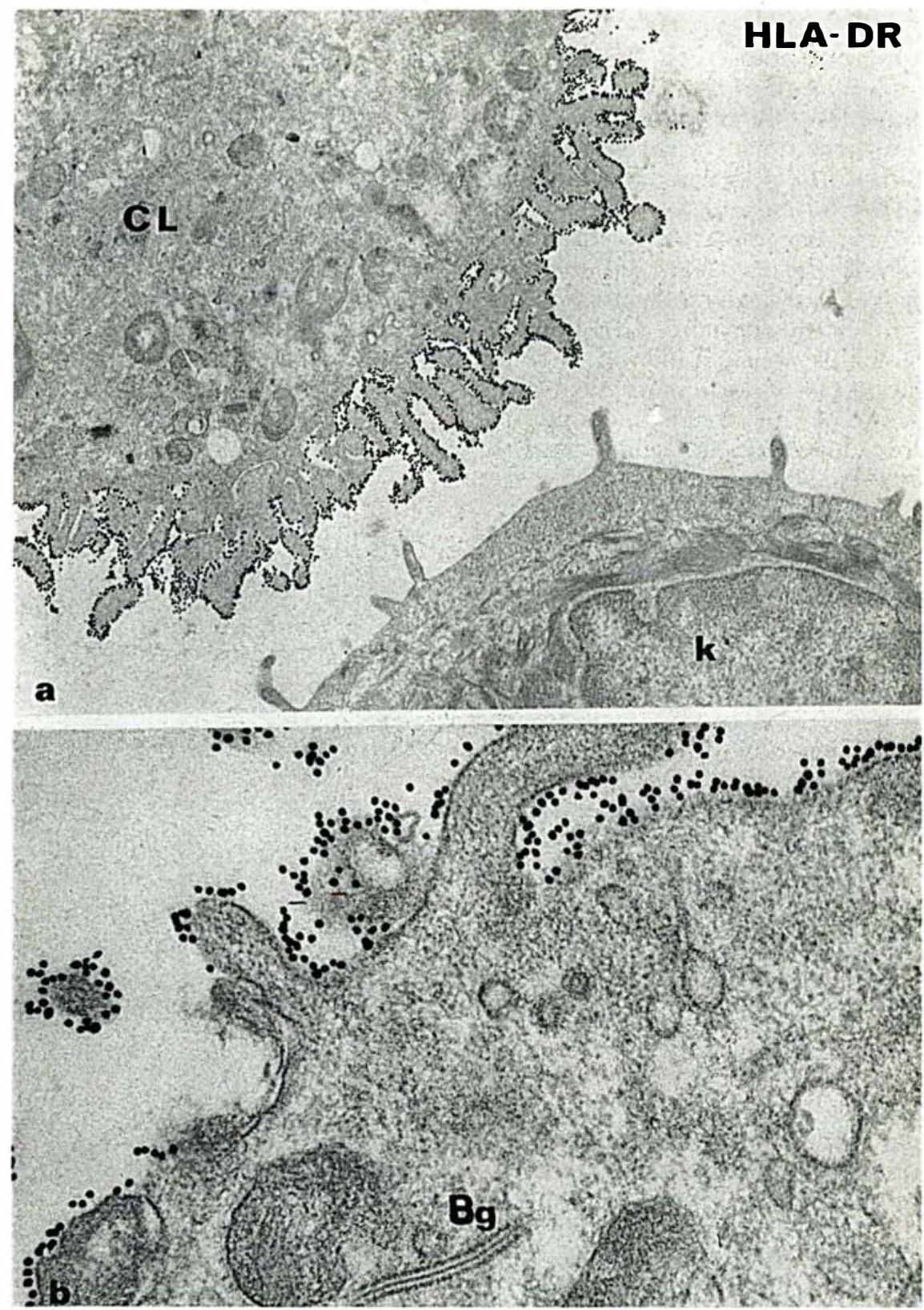

Figure 3. Identification immunomorphologique des antigènes HLA-DR exprimés à la surface des cellules de Langerhans (CL) par immunomarquage à l'or colloïdal. (a) Notez la densité très forte des grains d'or à la surface de la cellule de Langerhans et l'absence de marquage du kératinocyte (k). Cette cellule de Langerhans très fortement DR+ appartient à la sous-population exprimant dans l'épiderme normal cet antigène avec une forte intensité (voir texte); (b) détail du marquage de surface de la cellule de Langerhans caractérisée par la présence dans son cytoplasme d'un granule de Birbeck (Bg). (a $\times 13700$, b X 67000).

surface reste à préciser sur un plan fonctionnel.

\section{Endocytose et granules de Birbeck}

La capacité d'endocytose des cellules de Langerhans a fait l'objet de tra- vaux récents [14]. Le marquage des molécules $\mathrm{CDl}$ avec un anticorps spécifique sur cellules vivantes entraîne une endocytose du complexe $\mathrm{Ag}$-Ac, faisant intervenir des structures cytoplasmiques (vésicules recouvertes, endosomes, lysosomes, phagosomes) caractéristiques de l'en- 


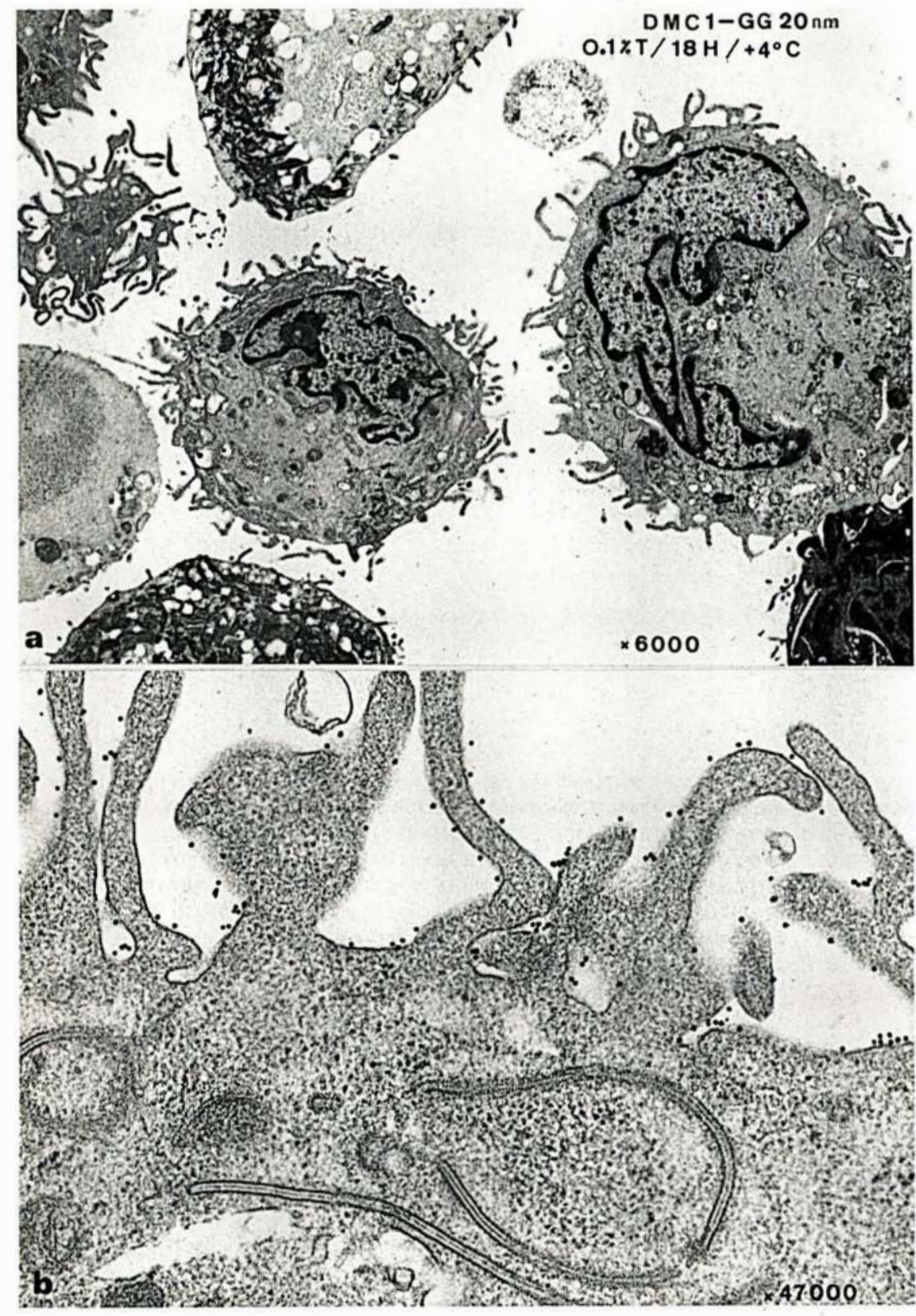

Figure 4. Les cellules de Langerhans expriment les antigènes CD1a et CD1c. L'anticorps monoclonal DMC1 reconnaît les molécules natives CD1a $(49 \mathrm{kDa}$ ). (a) Suspension de cellules épidermiques enrichie en cellules de Langerhans (4 cellules dendritiques) obtenue par action de la trypsine $10,1 \%$ pendant 18 heures $a+4^{\circ} \mathrm{C}$ ). L'anticorps DMC1 est directement conjugué avec des grains d'or de $20 \mathrm{~nm}$; (b) détail de l'identification des antigènes CD1a exprimés sur la membrane de la cellule de Langerhans par l'anticorps DMC1. Notez la présence de granules de Birbeck de grande taille. $(a \times 6000, b \times 47000)$

docytose des complexes de types récepteur-ligand.

Les molécules HLA-DR et CD4 suivent le même processus lorsqu'elles sont complexées avec une immunoglobuline spécifique. Ce phénomène $\mathrm{m} / \mathrm{s} n^{\circ} 2$ vol. 5 , férier 89 cellule de Langerhans. Cette endocytose mime ce qui se passe lors de la captation et de l'endocytose d'un antigène exogène avant la présentation antigénique aux lymphocytes [15].

En 1961, Birbeck [16] décrit dans les cellules de Langerhans les formations cytoplasmiques qui portent son nom (figure 5, p. 108). Ces formations correspondant à un accolement de deux portions de membrane ont une origine membranaire et peuvent être associées à des vacuoles, le plus souvent électroniquement vides, qui donnent un aspect en raquette Le nombre (très variable) et la taille (longueur, donc surface d'accolement) ne sont reliés à aucun autre paramètre mesurable de la cellule.

Lors des processus d'endocytose, les molécules impliquées ( $\mathrm{DR}, \mathrm{CDl}$ CD4) sont retrouvées au niveau de la ligne centrale des granules de Birbeck liés ou proches de la membrane cellulaire, mais sans concentration notable (figure 6, p. 109).

Les granules de Birbeck liés au processus d'endocytose des cellules de Langerhans sont un des éléments des phénomènes généraux touchant la dynamique membranaire mais sans spécificité apparente vis-à-vis d'un antigène donné. Ils représentent une voie d'adaptation de la cellule dans le processus lui permettant de produire ou d'endocyter rapidement une surface membranaire importante. Ceci peut être rapproché de la localisation très spécifique de cette cellule dendritique au niveau du revêtement cutanéo-muqueux où la densité des exo-antigènes est par définition maximale.

Ceci est également à rapprocher du fait que les granules de Birbeck semblent spécifiquement liés à un environnement épithélial.

In vitro [17], il est possible d'induire dans les cellules de Langerhans des structures de type granules de Birbeck en utilisant des anticorps ou des détergents. La nature biochimique de ces formations reste à déterminer.

\section{Cellules de Langerhans et présentation antigénique} on observe ensuite un recyclage des molécules endocytées à la surface de la cellule, dans un délai bref (une heure) et d'une manière indépendante de la capacité de synthèse de la
Les cellules de Langerhans font partie des cellules dendritiques capables de présenter les antigènes aux lym- 

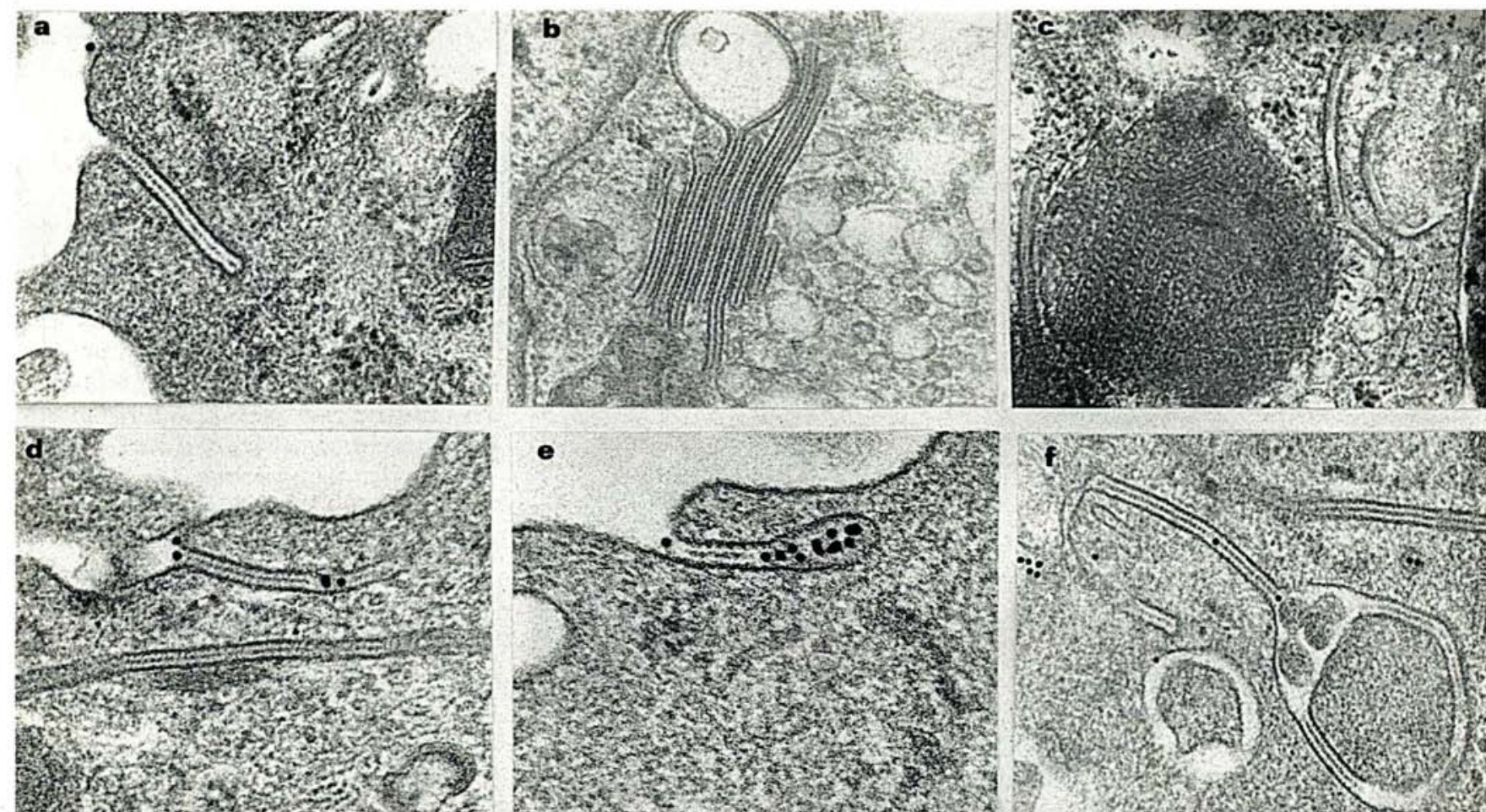

Figure 5. Les granules de Birbeck sont des organites spécifiques de la cellule de Langerhans. (a) Ils peuvent se former par accolement au niveau de la membrane cellulaire; (b) leur taille et leur nombre varient. On distingue des formes en bâtonnets ou en raquettes; (c) lorsque le plan de coupe passe de façon trangentielle par la ligne médiane du granule de Birbeck, on observe une structure pseudo-cristalline. Les granules de Birbeck sont impliqués dans l'endocytose d'antigènes exogènes ou de complexes immuns fixés par la cellule de Langerhans; (d) formation membranaire d'un granule de Birbeck contenant des complexes Ag CD1-Ac anti-CD1-Ac anti-lg couplés avec des grains d'or; (e) même image de formation membranaire d'un granule de Birbeck contenant des complexes immuns marqués et en relation avec une vacuole d'endocytose; (f) aspect cytoplasmique des granules de Birbeck, qui peuvent former des structures tridimentionnelles complexes. (a $\times 75000, b \times 67000, c \times 82000, d \times 75000, e \times 95000, f \times 75000)$.

phocytes (figure $7, p .111$ ) et d'induire une réponse immune de type cellulaire (hypersensibilité retardée) $[18,19]$.

Les conditions de cette stimulation sont relativement définies: a) le contact cellule de Langerhans-lymphocytes est indispensable ; les anticorps anti-HLA-DR bloquent cette fonction; b) dans le modèle de la culture mixte cellule de Langerhanslymphocytes allogéniques [20], ce sont les molécules HLA de classe II (et plus particulièrement les déterminants polymorphiques) qui sont reconnus par le récepteur des cellules T. In vivo, on admet que c'est le complexe antigène-molécules de classe II qui est présenté et reconnu au niveau des récepteurs $T$.

En système autologue, la stimulation des lymphocytes par les cellules expérimentalement (antigène soluble $\mathrm{PPD}^{*}$ ou antigène tuberculinique, ovalbumine ou haptènes), lors de modifications microbiennes ou virales (virus de l'herpès), ou lors de la sensibilisation (sulfate de nickel) des cellules épidermiques.

Les cellules dde Langerhans sont nécessaires à la production de cellules $\mathrm{T}$ cytotoxiques spécifiques des cellules épidermiques modifiées par un haptène [21, 22].

Dans certaines situations pathologiques ou in vitro, sous l'influence de certaines lymphokines (interféron $\gamma$ ), les kératinocytes expriment les molécules HLA de classe II. Fonctionnellement, ces kératinocytes, en l'absence de cellules de Langerhans, sont cependant incapables d'induire une prolifération lymphocytaire.

* PPD : protéine purifiée tuberculinique.

\section{Pharmacologie et cellules de Langerhans}

Les cellules de Langerhans produisent de l'interleukine l (Il-1), essentielle à l'activation des lymphocytes T. Elles sont également capables de produire les prostaglandines D2 intervenant dans les réactions inflammatoires [23].

Les ultraviolets $\mathrm{B}$ à forte dose réduisent le nombre des cellules de Langerhans chez l'homme. Il ne semble pas exister de modifications du nombre des cellules de Langerhans avec le vieillissement.

La PUVA thérapie diminue la densité des cellules de Langerhans dans le psoriasis. In vitro, la PUVA**

* PUV.4 : association psoralènes photosensibilisants et irradiation par UVA (ultraviolets A). 
diminue la capacité de stimulation des cellules de Langerhans en culture mixte allogénique.

Les rétinoïdes agissent sur les cellules de Langerhans: augmentation de la densité épidermique dans un premier temps, puis réduction avant de revenir à une valeur normale chez la souris. On doit retenir d'une façon générale un effet de réduction sur le nombre des cellules de Langerhans.

Chez la souris, le MBEH (monobenzyl-éther d'hydroquinone, qui est un sensibilisant) entraîne localement une augmentation du nombre des cellules de Langerhans.

Rappelons ici que l'interféron $\gamma$ ou la maintenance in vitro pendant 24 heures entraîne une forte augmentation de la densité des antigènes HLA-DR au niveau des cellules de Langerhans normales.

Dans ce domaine, la difficulté majeure provient du fait qu'il est difficile d'obtenir des suspensions cellulaires pures (plus de $98 \%$ de CL) et que, in vitro, ces cellules de Langerhans ne se divisent pas et perdent leurs caractères spécifiques.

Dans le futur, le rôle de différents facteurs - interleukines, GM-CSF (colony stimulating factor, spécifique de la lignée granulomonocytaire), interférons, drogues immunomodulatrices - sera une voie de recherche à creuser.

L'exemple de la ciclosporine A, qui ne modifie ni le nombre ni la localisation des cellules de Langerhans, mais qui affecte leur capacité de présentation antigénique chez la souris, mérite d'être développé.

\section{Cellules de Langerhans et pathologie cutanée}

Peu de données nouvelles ont été mises en évidence dans ce domaine en dehors du SIDA [24].

Classiquement, les cellules de Langerhans sont impliquées dans la dermite de contact où elles jouent un rôle essentiel dans le déclenchement de la réaction immune de type hypersensibilité retardée. Morphologiquement, on note des modifications des cellules de Langerhans (nombre, dendrites, signes cytoplasmiques d'activation). Certains auteurs ont pu parler de «cellules cibles » à propos des cellules de Langerhans dans cette affection, sur la base de données $\mathrm{m} / \mathrm{s} n^{\circ} 2$ vol. 5 , féurier 89 ultrastructurales. Cette interprétation reste critiquée.

Dans la dermatite atopique, la mise en évidence de récepteurs pour les $\mathrm{IgE}$ suggère que ces molécules interviennent dans la fixation des allergènes par les cellules de Langerhans. Dans les lymphomes épidermotropes, la notion essentielle est l'augmentation du nombre et la localisation épidermique et dermique des cellules de Langerhans, souvent en contact étroit avec les lymphocytes infiltrant derme et épiderme. Là encore les cellules de Langerhans ont été parfois considérés comme les «cellules cibles», ce qui n'est pas vérifié

Dans l'histiocytose $\mathrm{X}$, les cellules de Langerhans prolifèrent et présentent tous les marqueurs des cellules de Langerhans normales. Les antigènes $\mathrm{CDl}$ restent un élément majeur de confirmation du diagnostic avant la présence de granules de Birbeck dont la densité par cellule et la répartition cytoplasmique varient. Récemment, des cellules d'histiocytose X ont été utilisées pour immuniser des souris et produire un anticorps monoclonal
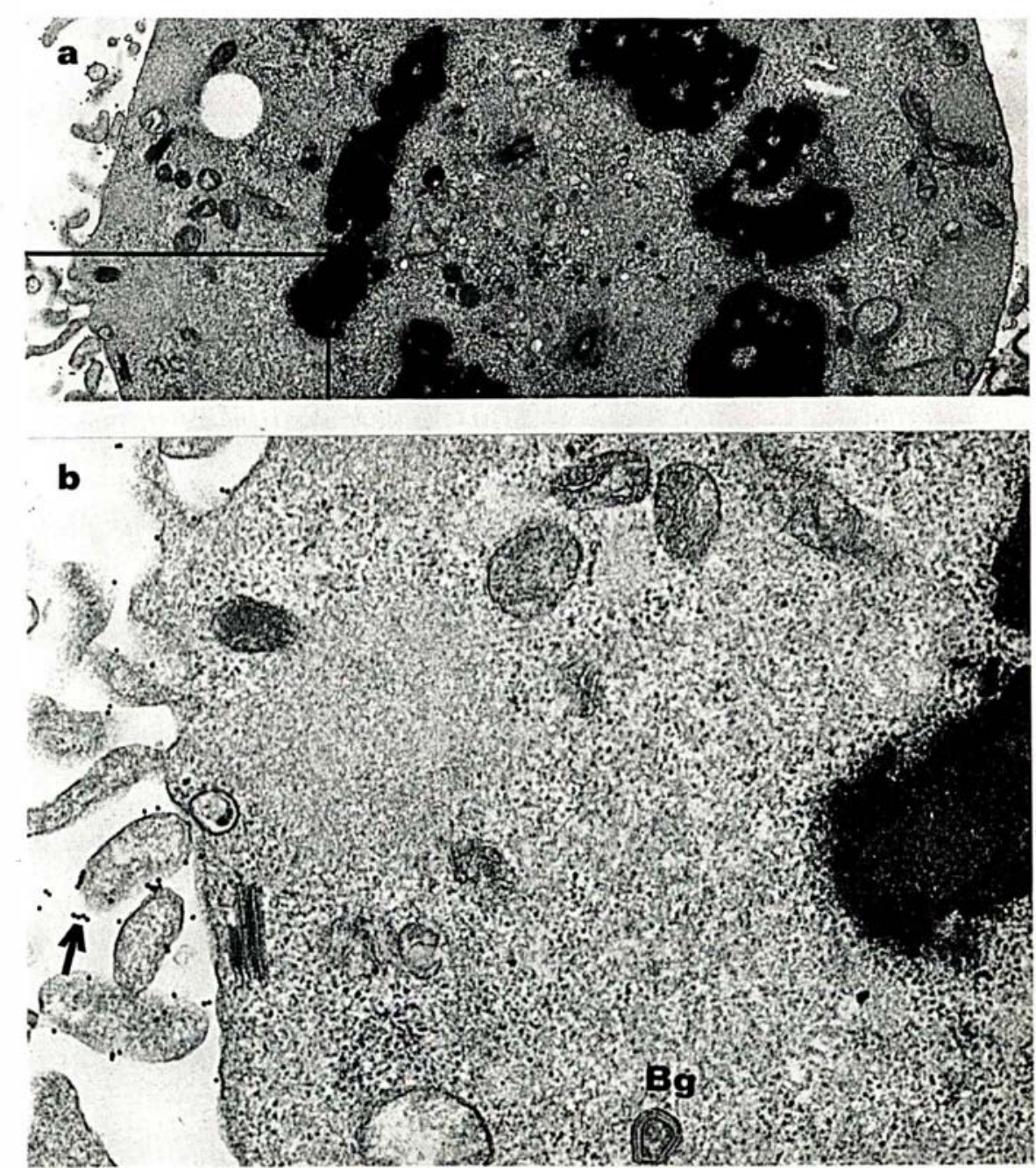

Figure 6. Les cellules de Langerhans peuvent se diviser au niveau de l'épiderme. (a) Cellule de Langerhans en mitose (chromosomes visibles) observée dans une suspension de cellules épidermiques normales; (b) détail de l'encart de la figure 6a montrant les granules de Birbeck (Bg) et les antigènes CD1a à la surface de la cellule (flèche) identifiés par immunomarquage à l'or colloïdal. (a $X$ $14000, b \times 32000)$. 


\section{RÉFÉRENCES}

21. Pehamberger $\mathrm{H}$, Stingl LA, Pogantsch $\mathrm{S}$, Steiner G, Wolff K, Stingl G. Epidermal cellinduced generation of cytotoxic $T$ lymphocyte responses against alloantigens or TNP modified syngenic celles : requirement of Iapositive Langerhans cells. J Invest Dermatol $1983 ; 81: 208-11$.

22. Faure M, Frappaz A, Schmitt D, DezutterDambuyant C, Thivolet J. Role of HLA-DR bearing Langerhans cells and epidermal indeterminate cells in the in vitro generation of alloreactive cytotoxic $\mathrm{T}$ cells in man. Cel Imınunol 1984; 83 : 271-9.

23. Czernielewski J. Modification of Langerhans cell population produced by differen pharmacological agents. In : Thivolet J Schmitt D, eds. The Langerhans Cell Inserm/ John Libbey Eurotext, 1988 : 217-24.

24. Haftek M. Langerhans cells in cutaneous pathology. In : Thivolet J, Schmitt D, eds. Inserm/John Libbey Eurotext, 1988 : 377-85.

25. Chardonnet Y, Viac J, Thivolet J Langerhans cells in human warts lesions. $\mathrm{Br}$ J Dermatol 1986 ; 115 : 669-75.

26. Belsito D, Sanchez M, Baer R, Valentine F, Torbecke G. Reduced Langerhans cell Ia antigen and ATP ase activity in patient with acquired immunodeficiency syndrome. $N$ Engl J Med 1984; 310 : 1279-82.

27. Tschachler E, Groh V, Popovic, et al. Epidermal Langerhans cells. A target for HTLV III/LAV infection. J Invest Dermatol $1987 ; 88: 233-7$

28. Gielen V, Schmitt D, Dezutter-Dambuyant C, Thivolet J, AIDS and Langerhans cells : CD4 antigenic site density modification evidenced by singel-cell immunogold labeling. Reg Immunol, (USA) 1989; 2 (sous presse).

29. Staquet MJ, Dezutter-Dambuyant C Zambruno G, Schmitt D, Thivolet J. Langerhans cells and basal keratinocytes express adhesive protein receptors related to the VLA family. In: Thivolet J, Schmitt D, eds. The Langerhans Cell Inserm/John Libbey Eurotext, $1988 ; 167-77$.

30. De Panfilis G, Manara G, Ferrari C, Torresani C, Donelli S, Caleffi E. Reactivity o Langerhans cells with anti-CDI lc monoclonal antibody. In : Thivolet J, Schmitt D, eds

The Langerhans Cell. Inserm/John Libbey appelé $\mathrm{DMCl}$ (une IgGl) réagissant avec les molécules CDla des cellules de Langerhans proliférantes ou normales et des thymocytes.

Dans les lésions cutanées présentant un infiltrat cellulaire lympho-histiocytaire, il semble que les cellules de Langerhans soient impliquées (sur la base de données essentiellement morphologiques). Une attention particulière a été portée au psoriasis et au lichen plan.

Les cellules de Langerhans ont été récemment reconsidérées dans certaines lésions virales ou liées à une infection virale. Ainsi, dans les papillomes [25], il apparaît des modifications de la densité des cellules de Langerhans, au niveau des lésions cutanées ou muqueuses. Le VPH (virus du papillome humain) n'a jamais été trouvé dans les cellules de Langerhans.

Dans le SIDA (virus HIV) ont été observés : (a) une diminution de la densité des cellules de Langerhans au cours du développement de la-maladie [26]; (b) une infection directe des cellules de Langerhans par le virus HIV (présence de protéines virales, images de bourgeonnements viraux, particules virales entières in situ dans l'épiderme [27], capacité des cellules épidermiques de patients séropositifs d'infecter in vitro des cellules monocytaires normales); (c) une disparition des cellules de Langerhans dans les lésions de Kaposi*.

Cette infection des cellules de Langerhans est à rapprocher de l'existence des molécules CD4 [27] à la surface de ces cellules, molécules considérées comme des récepteurs du virus. Pourtant, il apparaît que cette infection des cellules de Langerhans est difficile à mettre en évidence (particules très rares). Ainsi les cellules de Langerhans doivent avant tout être considérées, dans le SIDA, comme des cellules-réservoirs potentiels du virus.

Leur capacité de fixer les protéines gpl20 d'enveloppe du HIV-l sont actuellement une voie de recherche dans le domaine des essais de vaccination par des protéines virales obtenues par génie génétique.

\footnotetext{
* Sur l'origine possible du Sarcome de Kaposi, voir m/s $n^{\circ} 1$, vol. 5 , page 61 et Science, numéro du 13 janvier 1989, vol.243,
} page 223.

\section{Conclusions}

Acteur principal de l'immunité de la peau, la cellule de Langerhans reste un terrain de recherche du fait de divers atouts: (a) sa localisation cutanée, qui permet une obtention relativement simple; (b) la possibilité de préparer à partir de la peau des suspensions fortement enrichies. en cellules de Langerhans (jusqu'à $80 \%$ de CL vivantes non marquées) ; (c) l'existence de tests fonctionnels in vitro (culture mixte lymphocytes-cellules épidermiques); (d) la possibilité de disposer d'un choix très large d'anticorps monoclonaux réagissant avec cette cellule ; $(e)$ une adaptation de plus en plus fine des méthodes de laboratoire à ce type cellulaire.

Elle est considérée maintenant comme un modèle des cellules présentant les antigènes - parmi l'ensemble des cellules dendritiques qui ont cette fonction.

L'un des facteurs limitants reste l'incapacité de les cultiver in vitro et même de maintenir in vitro leur caractères de différenciation (antigènes de surface et granules de Birbeck).

In vivo, le cycle des cellules de Langerhans (de la moelle à l'épiderme et de l'épiderme aux ganglions) et le devenir de la population périphérique (augmentation ou diminution) suggèrent l'existence d'un flux cellulaire modulable localement et dans le temps, pour lequel on connaît encore très mal les étapes extrêmes (médullaire et ganglionnaire) et dont la cellule de Langerhans épidermique «classique» ne représent que la phase intermédiaire. La phase finale de maturation des cellules de Langerhans (de l'épiderme aux ganglions) où doivent intervenir molécules d'adhésion [29, 30], matrice extracellulaire, cytokines, et le rôle précis des granules de Birbeck (dans le traitement de l'antigène ou antigen processing) sont des voies de recherche du plus haut intérêt.

La présence des cellules de Langerhans dans tous les épithéliums de revêtement (peau; muqueuses orale, anale, vaginale et pulmonaire ; vessie) renforce encore l'intérêt croissant qu'elles suscitent en dermatologie mais également en immunologie générale au titre des systèmes immunitaires périphériques 


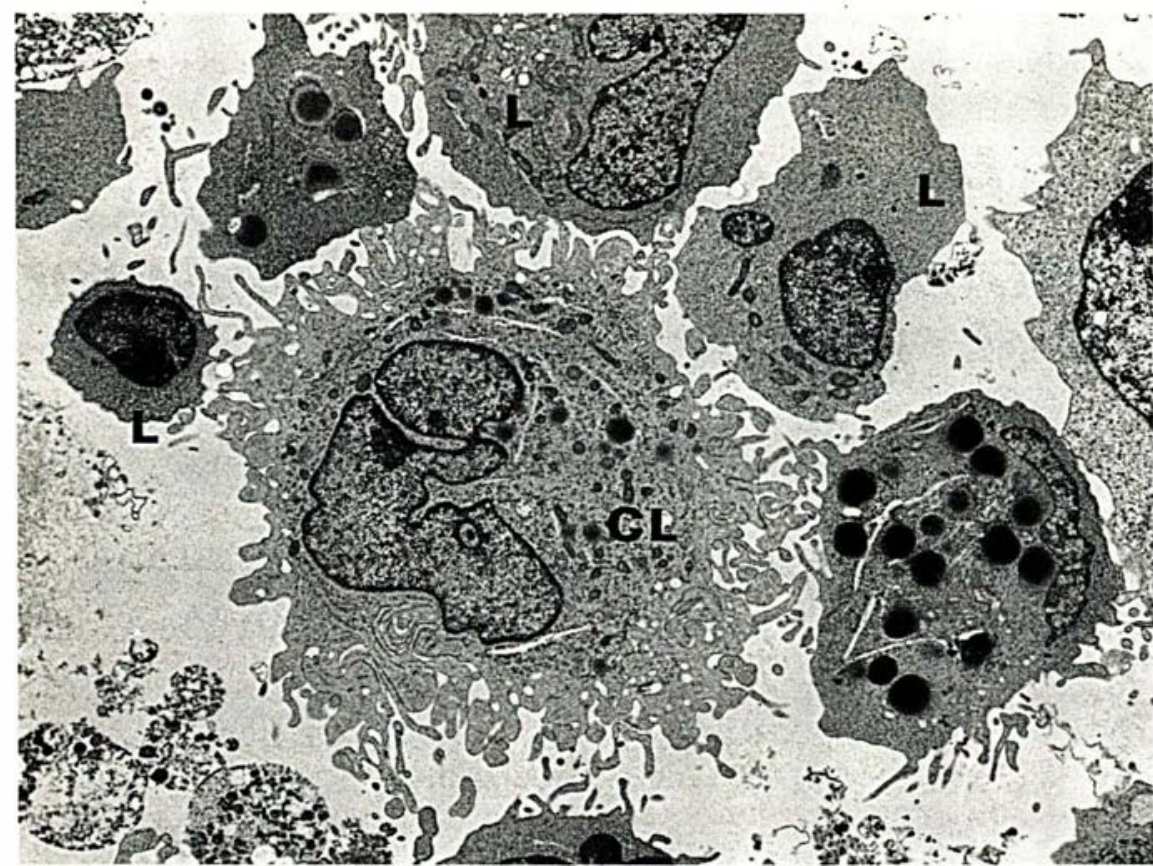

Figure 7. Les cellules de Langerhans présentent les antigènes aux lymphocytes $T$ auxiliaires. Cette présentation nécessite un contact direct. Le modèle de la culture mixte cellules épidermiques (contenant des cellules de Langerhans)lymphocytes allogéniques permet d'observer cette phase de présentation. Dans ces cultures mixtes, on observe des images de rosettes /ymphocytaires autour de cellules de Langerhans (CL). On note aussi des contacts membranaires étroits entre les $C L$ et les lymphocytes (L). ( $\times 6000)$.

\section{Summary}

The Langerhans cell : the dendritic cell of skin and mucosa

The Langerhans cells are the immunocompetent dendritic cells in the epidermis and the stratified epithelia (oral, anal and vaginal mucosa, bladder epithelium, bronchial and corneal epithela). These cells represent a subpopulation of the mononuclear phagocyte system and they play a great role in the induction of the immune response. Langerhans cells are able to catch exogeneous antigens and to present them to helper T lymphocytes. As antigen presenting cells they are able to induce $\mathrm{T}$ cell proliferation. Although they are in low percentage in the normal epidermis $(2 \%)$ they play a central role in the infor- mation of the lymphoid system about the foreign antigens entering the body through the epithelia. In human pathology the Langerhans cells are involved in the process of graft rejection, anti-tumoral inflammatory reactions, contact dermatitis and psoriasis. They are present in bronchial epithelium which is a privileged place for antigen penetration and immunoallergic reactions. Furthermore, they express the membrane CD4 molecules which are considered as receptors for Human Immunodeficiency Virus (HIV) (virus of AIDS) and could be considered as a potential virus reservoir.
Professeur Pierre H. Cartier

Ancien responsable des laboratoires universitaire et hospitalier (B) de Biochimie du CHU Necker-Enfants Malades, et ancien directeur de l'Unité 75 de l'Inserm, le Professeur Pierre H. Cartier est décédé le 12 décembre 1988. Son activité scientifique s'était d'abord attachée à l'élucidation des processus biochimiques de l'ossification, dont il avait exposé la théorie ATPasique dans sa thèse de Sciences soutenue en 1952. Par la suite, les travaux de son équipe se sont orientés vers l'étude du métabolisme énergétique du globule rouge normal ou pathologique, et de sa régulation: recherche du retentissement métabolique de divers déficits enzymatiques, de la conservation du sang, de modifications du $\mathrm{pH}$; étude plus spécifique de la pyruvate kinase érythrocytaire, dont le caractère allostérique fut démontré (1968). L'investigation du métabolisme des purines et des pyrimidines, en relation avec les hyperuricémies et les déficits immunitaires congénitaux, est également devenue une des orientations majeures de son laboratoire, et il a décrit, en 1974, le premier cas mondial de déficit en adénine phosphoribosyltransférase responsable d'une lithiase urinaire composée de 2,8-dihydroxyadénine. 